\title{
EDITORIAL
}

\section{Chronic Obstructive Pulmonary Disease with Sleep-related Hypoxemia: Nocturnal Oxygen is not the Answer}

\author{
Tejas M Suri ${ }^{1}$, Jagdish C Suri ${ }^{2}$ \\ Indian Journal of Sleep Medicine (2021): 10.5005/jp-journals-10069-0074
}

Long-term supplemental oxygen therapy (LTOT) provided in a domiciliary setting has been shown to improve survival among patients with chronic obstructive pulmonary disease (COPD) who have severe daytime resting hypoxemia. ${ }^{1,2}$ This encompasses patients with arterial oxygen partial pressure $\left(\mathrm{PaO}_{2}\right)$ of less than $56 \mathrm{~mm} \mathrm{Hg}$ (or oxygen saturation $\left(\mathrm{SpO}_{2}\right)$ less than $88 \%$ ) and those with $\mathrm{PaO}_{2}$ of $56-59 \mathrm{~mm} \mathrm{Hg}$ with polycythemia or cor pulmonale. However, a significant proportion of COPD patients without severe daytime hypoxemia have nocturnal oxygen desaturation. Significant nocturnal desaturation refers to an $\mathrm{SpO}_{2}$ of less than $90 \%$ more than $30 \%$ of the night. ${ }^{3}$ In a study of 128 patients with mild-to-moderate daytime hypoxemia $\left(\mathrm{PaO}_{2}\right.$ between $56 \mathrm{~mm} \mathrm{Hg}$ and $69 \mathrm{~mm} \mathrm{Hg}$ ), nocturnal desaturation was observed in $54 \%$. About one-third of these had cyclical desaturations suggestive of obstructive sleep apnea (OSA), whereas two-thirds had sustained nonapneic hypoxemia. ${ }^{4}$ Nocturnal oxygen desaturation is associated with cardiac arrhythmias and an increase in pulmonary artery pressure. ${ }^{5}$ It is also associated with a rise in inflammatory markers such as high-sensitivity C-reactive protein (hs-CRP) that increases risk of cardiovascular disease. ${ }^{6}$ Finally, nocturnal oxygen desaturation in COPD is associated with progression of respiratory failure to requirement for LTOT. ${ }^{7}$

While the overlap syndrome of OSA and COPD is treated with positive airway pressure (PAP) therapy, the optimal treatment for isolated, nonapneic nocturnal hypoxemia in COPD is unknown. Three randomized controlled trials have studied the role of nocturnal oxygen supplementation (targeting $\mathrm{SpO}_{2}$ greater than 90\%) in these patients. ${ }^{8-10}$ These trials have failed to show any benefit of nocturnal oxygen therapy in improving survival or preventing progression to LTOT requirement. Although the trials individually had small sample sizes, they collectively conclude that nocturnal oxygen is not beneficial in COPD patients with isolated, nonapneic nocturnal hypoxemia.

In view of the adverse consequences of nocturnal desaturation in COPD, it is important to continue the search for appropriate therapies for these patients. In this regard, it is helpful to revisit the pathophysiology of nocturnal hypoxemia in COPD. Sleep is associated with decreased wakefulness drive to the respiratory system, blunting of hypoxemic and hypercapnic ventilatory responses, and decreased pharyngeal dilator muscle activity with upper airway narrowing. ${ }^{11}$ These changes lead to reduced minute ventilation during the sleep. While these changes are inconsequential in healthy individuals, COPD patients with preexisting mild-to-moderate hypoxemia due to ventilation-perfusion mismatch experience precipitous drops in $\mathrm{SpO}_{2}$ during the sleep. Further, COPD patients with emphysema have downward displacement of the diaphragm
${ }^{1}$ Pulmonary, Critical Care and Sleep Medicine, All India Institute of Medical Sciences, New Delhi, India

${ }^{2}$ Pulmonary, Critical Care and Sleep Medicine, Fortis Flt Lt Rajan Dhall Hospital, Vasant Kunj, New Delhi, India

Corresponding Author: Jagdish C Suri, Pulmonary, Critical Care and Sleep Medicine, Fortis Flt Lt Rajan Dhall Hospital, Vasant Kunj, New Delhi, India, Phone: +91 9810097259, e-mail: docjcsuri@ gmail.com

How to cite this article: Suri TM, Suri JC. Chronic Obstructive Pulmonary Disease with Sleep-related Hypoxemia: Nocturnal Oxygen is not the Answer. J Sleep Med 2021;16(2):19-20.

Source of support: Nil

Conflict of interest: None

that places it at a mechanical disadvantage. These patients are reliant on accessory muscles to supplement their respiratory efforts. Rapid eye movement (REM) sleep results in atonia of the accessory muscles leading to hypoventilation and REM-related desaturations. ${ }^{11}$

Although the aforementioned randomized trials of oxygen supplementation in COPD with nocturnal desaturation excluded OSA patients, they did not evaluate patients for sleep-related hypoventilation prior to enrollment. Further, these trials did not exclude patients with daytime hypercapnia. ${ }^{8-10} \mathrm{~A}$ recent metaanalysis has found that domiciliary noninvasive ventilation (NIV) may improve survival in COPD patients with chronic daytime hypercapnia. ${ }^{12}$ Based on this, the American Thoracic Society (ATS) has recommended home NIV therapy in COPD patients with awake $\mathrm{PaCO}_{2}$ greater than $45 \mathrm{~mm} \mathrm{Hg}{ }^{12}$ Sleep-related hypoventilation (defined as a rise in arterial partial pressure of carbon dioxide $\left(\mathrm{PaCO}_{2}\right)$ by $10 \mathrm{~mm} \mathrm{Hg}$ (or $>45 \mathrm{~mm} \mathrm{Hg}$ ) for 10 minutes during the sleep) may be a precursor of chronic daytime hypercapnia in COPD patients. ${ }^{13}$ Hence, clinical trials of nocturnal NIV in these patients to prevent the progression of respiratory failure and mortality are warranted.

In conclusion, nocturnal oxygen desaturations are common among COPD patients with mild-to-moderate hypoxemia. The nocturnal hypoxemia may be a consequence of OSA or sleeprelated hypoventilation. These patients may be considered for polysomnography with transcutaneous capnometry. Those who are diagnosed with COPD-OSA overlap have PAP titration performed and are treated with PAP therapy. Supplemental oxygen is not useful for nonapneic nocturnal hypoxemia and the optimal therapy for these patients is unknown. However, these patients may be evaluated for sleep-related hypoventilation. Clinical trials of NIV therapy are of utmost necessity in COPD 
patients with nocturnal hypoxemia due to sleep-related hypoventilation.

\section{References}

1. Continuous or nocturnal oxygen therapy in hypoxemic chronic obstructive lung disease: a clinical trial. Nocturnal Oxygen Therapy Trial Group. Ann Intern Med 1980;93(3):391-398. DOI: 10.7326/00034819-93-3-391.

2. Long term domiciliary oxygen therapy in chronic hypoxic cor pulmonale complicating chronic bronchitis and emphysema. Report of the Medical Research Council Working Party. Lancet Lond Engl 1981;1(8222):681-686. DOI: 10.1016/S0140-6736(81)91970-X

3. Lewis $C A$, Fergusson W, Eaton T, et al. Isolated nocturnal desaturation in COPD: prevalence and impact on quality of life and sleep. Thorax 2009;64(2):133-138. DOI: 10.1136/thx.2007.088930.

4. Lacasse $Y$, Sériès $F$, Vujovic-Zotovic $N$, et al. Evaluating nocturnal oxygen desaturation in COPD--revised. Respir Med 2011;105(9):13311337. DOI: 10.1016/j.rmed.2011.04.003.

5. Lacasse Y, Bernard S, Sériès F, et al. Multi-center, randomized, placebocontrolled trial of nocturnal oxygen therapy in chronic obstructive pulmonary disease: a study protocol for the INOX trial. BMC Pulm Med 2017;17(1):8. DOI: 10.1186/s12890-016-0343-9.

6. Macrea MM, Owens RL, Martin T, et al. The effect of isolated nocturnal oxygen desaturations on serum hs-CRP and IL- 6 in patients with chronic obstructive pulmonary disease. Clin Respir J 2019;13(2):120 124. DOI: 10.1111/crj.12992.
7. Sergi M, Rizzi M, Andreoli A, et al. Are COPD patients with nocturnal REM sleep-related desaturations more prone to developing chronic respiratory failure requiring long-term oxygen therapy? Respir Int Rev Thorac Dis 2002;69(2):117-122. DOI: 10.1159/000056313.

8. Fletcher EC, Luckett RA, Goodnight-White $S$, et al. A double-blind trial of nocturnal supplemental oxygen for sleep desaturation in patients with chronic obstructive pulmonary disease and a daytime $\mathrm{PaO}_{2}$ above $60 \mathrm{~mm} \mathrm{Hg}$. Am Rev Respir Dis 1992;145(5):1070-1076. DOI: 10.1164/ajrccm/145.5.1070.

9. Chaouat $A$, Weitzenblum $E$, Kessler $R$, et al. A randomized trial of nocturnal oxygen therapy in chronic obstructive pulmonary disease patients. Eur Respir J 1999;14(5):1002-1008. DOI: 10.1183/09031936.99.14510029.

10. Lacasse $Y$, Sériès $F$, Corbeil F, et al. Randomized trial of nocturnal oxygen in chronic obstructive pulmonary disease. N Engl J Med 2020;383(12):1129-1138. DOI: 10.1056/NEJMoa2013219.

11. Suri TM, Suri JC. A review of therapies for the overlap syndrome of obstructive sleep apnea and chronic obstructive pulmonary disease. FASEB BioAdvances 2021. DOI: 10.1096/fba.2021-00024.

12. Macrea M, Oczkowski S, Rochwerg B, et al. Long-term noninvasive ventilation in chronic stable hypercapnic chronic obstructive pulmonary disease. An official American Thoracic Society clinical practice guideline. Am J Respir Crit Care Med 2020;202(4):e74-e87. DOI: 10.1164/rccm.202006-2382ST.

13. Böing S, Randerath WJ. Chronic hypoventilation syndromes and sleep-related hypoventilation. J Thorac Dis 2015;7(8):1273-1285. DOI: 10.3978/j.issn.2072-1439.2015.06.10. 